\title{
Internações por "Outras gravidezes que terminam em aborto". CID 00-008 na região metropolitana de Porto Alegre
}

\author{
Hospitalizations for "Other pregnances that end in abortion". CID 00-008 in the metropolitan region of Porto Alegre \\ Hospitalizaciones por "Otros embarazos que terminan en aborto". CID 00-008 en la región metropolitana de Porto Alegre
}

\begin{abstract}
RESUMO
O aborto tornou-se um problema de saúde pública a partir dos altos índices de morte obstétrica, decorrentes dos abortamentos clandestinos. Objetivo: caracterizar o perfil das hospitalizações por "outras gravidezes que terminaram em aborto" em Porto Alegre e sua Região Metropolitana entre 2016 e 2018. Método: Estudo epidemiológico de base populacional, observacional e transversal a partir do Sistema de Informações Hospitalares SIH/SUS. Resultados: No período de 2016 a 2018 foram registradas 3.640 internações por "outras gravidezes que terminaram em aborto" na região em estudo. As maiores taxas de hospitalizações ocorreram em Porto Alegre, Canoas e Alvorada, perfazendo 42,06\%, 10,47\% e 10,11\%, respectivamente. Quanto a faixa etária, a media variou entre 20 e 29 anos de idade. Conclusão: Se faz necessário aprofundar o conhecimento desta realidade na região estudada, como por exemplo, o motivo pelo qual os abortos foram causados e as condições sociodemográficas destas mulheres, que podem elucidar os fatores predisponentes.
\end{abstract}

DESCRITORES: Saúde Materna; Aborto; Saúde Pública.

\section{ABSTRACT}

Abortion has become a serious public health problem, due to the high rates of obstetric death resulting from clandestine abortions practiced by women of all social classes. Objective: to characterize the profile of hospitalizations for "other pregnancies that ended in abortion" in the Metropolitan Region of Porto Alegre between 2016 and 2018. Method: A population-based, observational and cross-sectional epidemiological study using the Hospital Information System SIH / SUS, available publicly. Results: In the period from 2016 to 2018, there were 3,640 hospitalizations for "other pregnancies that ended in abortion" in the Metropolitan Region of Porto Alegre. The highest rate of hospitalizations occurred in Porto Alegre, Canoas and Alvorada $(42.06 \%, 10.47 \%$ and $10.11 \%)$, respectively. As for the age group the majority of the women were between 20 and 29 years of age. Conclusion: It is necessary to deepen the knowledge of this reality in the region studied, for example, the reason abortions were caused and the sociodemographic conditions of these women that can elucidate the predisposing factors.

DESCRIPTORS: Maternal Health; Abortion; Public Health.

\section{RESUMEN}

El aborto se ha convertido en un grave problema de salud pública debido a las altas tasas de muerte obstétrica, producto de abortos clandestinos practicados por mujeres de todas las clases sociales. Objetivo: El estudio tuvo como objetivo caracterizar el perfil de hospitalizaciones por "otros embarazos que terminaron en aborto" en Porto Alegre y en la Región Metropolitana de Porto Alegre entre 2016 y 2018. Método: Estudio epidemiológico poblacional, observacional y transversal basado en el Sistema de Información Hospitalaria SIH / SUS, disponible públicamente. Resultados: En el período de 2016 a 2018, se registraron 3.640 hospitalizaciones por "otros embarazos que terminaron en aborto" en la región en estudio. Las mayores tasas de hospitalización se dieron en Porto Alegre, Canoas y Alvorada, con $42,06 \%, 10,47 \%$ y 10,11\%, respectivamente. En cuanto al grupo de edad, la media osciló entre los 20 y los 29 años. Conclusion: Es necesario profundizar en el conocimiento de esta realidad en la región estudiada, por ejemplo, la razón por la que se produjeron los abortos y las condiciones sociodemográficas de estas mujeres, lo que puede dilucidar los factores predisponentes.

DESCRIPTORES: Salud Maternal; Aborto; Salud Pública.

RECEBIDO EM: 15/08/2020 APROVADO EM: 27/08/2020 


\section{Kelly Cristina Fogazzi Rosso}

Enfermeira no Hospital Restinga e Extremo-Sul.

ORCID: 0000-0003-0553-1495

\section{Ana Lúcia Galbarino Amaral}

Enfermeira. Mediadora do Projeto ApiceON no Estado do Rio Grande do Sul. Mestranda em Ensino em Saúde na Universidade Federal de Ciências da Saúde de Porto Alegre.

ORCID: 0000-0001-5381-1681

\section{Luciana Medeiros Paungartner}

Enfermeira - Pós Graduação em UTI Geral e Gestão da Assistência Intensiva ao Paciente Crítico - Instituto Faveni .

ORCID: 0000-0003-1983-1114

\section{Amanda Pereira Ferreira Dellanhese}

Enfermeira. Doutora em Saúde da Criança e do Adolescente pela Universidade Federal do Rio Grande do Sul. Docente Colaboradora da Residência Integrada Multiprofissional em Saúde Coletiva da Universidade Federal do Rio Grande do Sul.

ORCID: 0000-0002-1515-9693

\section{Simone Lysakowski}

Doutoranda em Pediatria pela Universidade Federal de Ciências da Saúde de Porto Alegre (UFCSPA). Mestre em Ensino na Saude pela UFCSPA. Especialista em Docência no Ensino Superior pela Pontifícia Universidade Católica do Rio Grande do Sul (PUCRS). Especialista em Doação e Transplantes e em Oncologia pela Universidade São Camilo. Enfenrira na Organização de Procura de Órgãos (OPO 1) na Santa Casa de Misericórdia de Porto Alegre.

ORCID: 0000-0003-3959-956X.

\section{Morgana Thaís Carollo Fernandes}

Enfermeira. Pós-Doutoranda em Saúde da Criança pela Pontifícia Universidade Católica do Rio Grande do Sul (PUCRS), bolsista pela University of Toronto (UofT). Professora Colaboradora da Residência Multiprofissional em Saúde da Criança e Pesquisadora Associada do Programa de Extensão e Pesquisa em Saúde Urbana, Ambiente e Desigualdades da Universidade Federal do Rio Grande do Sul (UFRGS).

ORCID: 0000-0002-7989-294X

\section{INTRODUÇÃO}

0 aborto é uma das principais causas de morte materna no mundo, e sua maior incidência se dá nos países em desenvolvimento ${ }^{1}$. De acordo com a Organização Mundial de Saúde (OMS), anualmente ocorrem 22 milhões de abortamentos, que são cometidos de forma insegura, sendo que 47 mil mulheres vão a óbito por conta das complicações decorrentes ${ }^{2}$.

No Brasil o aborto inseguro causou a morte de 203 mulheres em 2016, e anualmente são notificados aproximadamente um milhão de abortamentos autoprovocados ${ }^{3}$. A Pesquisa Nacional de Aborto (PNA) aponta que uma em cada cinco mulheres em idade reprodutiva até os 40 anos, já interrompeu pelo menos uma gravidez ao longo de sua vida reprodutiva, perfazendo 4,7 milhões de abortos realizados em $2016^{3}$. O perfil epidemiológico de mortalidade das mulheres que abortam no Brasil é predominantemente de negras, jovens, solteiras que não concluíram o ensino fundamental ${ }^{3,4}$.

Acredita-se que as causas que levam as mulheres a tomarem essa decisão, possam estar relacionadas com a situação socioeconômica, falta de suporte do parceiro, violência doméstica, dificuldade de acesso a métodos contraceptivos e até mesmo falha na contracepção ${ }^{3,5}$. Mulheres em vulnerabilidade representam número expressivo de abortos, as histórias que contam não são tanto sobre o desejo de se livrar da gravidez, e sim sobre as condições sociais miseráveis que as fazem mudar de ideia sobre trazer novas vidas ao mundo ${ }^{6}$.

Em consequência da ilegalidade do abortamento no Brasil, mulheres buscam clínicas clandestinas com profissionais que se dizem capacitados para praticar o crime, podendo levar a infertilidade, infecção e grande número de óbitos ${ }^{7}$, além de tornar pouco conhecida a magnitude e repercussão do ato devido a subnotificação dos casos, impe- dindo a elaboração de indicadores confiáveis para implementação de políticas públicas eficientes ${ }^{8}$. A fragilidade na composição dos indicadores hospitalares acerca desta temática e a falta de normatização reforçam a problemática de saúde pública. A prevalência do aborto provocado ou induzido não é conhecida com exatidão, além disso, não existe um Código Internacional de Doença (CID) ou uma categoria restrita para aborto provocado ou induzido. No Departamento de Informática do Sistema Único de Saúde (DATASUS), além do CID-10 aborto espontâneo e aborto por razões médicas, está disponível a categoria "outras gravidezes que terminam em aborto" que deve ser integrado para contabilização estatística ${ }^{9,10}$.

Desse modo, o objetivo dessa pesquisa foi caracterizar o perfil das hospitalizações pelo CID "outras gravidezes que terminam em aborto" na Região Metropolitana de Porto Alegre (RMPA), entre os anos de 2016 a 2018. 


\section{METODOLOGIA}

Trata-se de um estudo epidemiológico de base populacional, observacional e transversal por meio de consulta aos arquivos

públicos do Sistema de Informações Hospitalares / Sistema Único de Saúde (SIH/ SUS) disponíveis no site www.datasus.saude.gov.br no formato RD5. A conferência foi feita pelo tabulador disponibilizado

Tabela 1- Internação por "outras gravidezes que terminam em aborto" em Porto Alegre e RMPA - RS de 2016 a 2018

\begin{tabular}{|c|c|c|}
\hline Município & Internações & $\%$ \\
\hline Porto Alegre & 1531 & 42,06 \\
\hline Canoas & 381 & 10,47 \\
\hline Alvorada & 368 & 10,11 \\
\hline Cachoeirinha & 343 & 9,42 \\
\hline São Leopoldo & 186 & 5,11 \\
\hline Esteio & 124 & 3,41 \\
\hline Parobé & 97 & 2,66 \\
\hline Sapucaia do Sul & 97 & 2,66 \\
\hline Montenegro & 78 & 2,14 \\
\hline Taquara & 69 & 1,90 \\
\hline São Jerônimo & 65 & 1,79 \\
\hline Novo Hamburgo & 60 & 1,65 \\
\hline Campo Bom & 55 & 1,51 \\
\hline Sapiranga & 53 & 1,46 \\
\hline Viamão & 45 & 1,24 \\
\hline Gravataí & 40 & 1,10 \\
\hline Dois Irmãos & 18 & 0,49 \\
\hline Estância Velha & 17 & 0,47 \\
\hline Triunfo & 10 & 0,27 \\
\hline Igrejinha & 2 & 0,05 \\
\hline Rolante & 1 & 0,03 \\
\hline Total & 3640 & $100 \%$ \\
\hline
\end{tabular}

Tabela 2 - Faixa etária das internações por "outras gravidezes que terminam em aborto" em Porto Alegre e RMPA - RS de 2016 a 2018

$\begin{array}{lcc}\quad \text { Faixa Etária } & \text { Internações } & \% \\ 10 \text { a } 14 \text { anos } & 23 & 0,63 \\ 15 \text { a } 19 \text { anos } & 400 & 10,99 \\ 20 \text { a } 29 \text { anos } & 1507 & 41,40 \\ 30 \text { a } 39 \text { anos } & 1361 & 37,39 \\ 40 \text { a } 49 \text { anos } & 343 & 9,42 \\ 50 \text { a } 59 \text { anos } & 4 & 0,17 \\ \text { Total } & 3638 & 100 \% \\ \text { Fonte: Ministério da Saúde/SVS - DATASUS. Elaborado pelos autores. } & \end{array}$

pelo Ministério da Saúde (MS) TabNET, que realiza cruzamentos de variáveis básicas diretamente na internet. Posteriormente os dados foram tratados com o tabulador $\mathrm{Ta}$ bWIN que permitiu tabulações mais avançadas sob os arquivos capturados.

Foram obtidos dados sociodemográficos das internações de mulheres residentes na capital e RMPA, no período de 2016 a 2018, com diagnóstico principal de outras gravidezes que terminam em aborto, CID 00-008. Como critério de inclusão, foram selecionados todos os casos referentes ao CID no período em estudo, disponibilizados pelo SIH.

Quanto aos aspectos éticos, os arquivos do DATASUS são de domínio público e disponíveis na internet. De acordo com a Resolução 466/2012 do Conselho Nacional de Saúde (CNS), o estudo não está, portanto, sujeito a avaliação do Comitê de Ética em Pesquisa/Comissão Nacional de Ética em Pesquisa (CEP/CONEP). Caracterizou-se o perfil das hospitalizações das mulheres por faixa etária (sem filtro de idade) e raça/cor além do caráter de internação, dias de permanência, gastos e óbitos ${ }^{11}$.

\section{RESULTADOS}

No período entre 2016 e 2018, foram registradas 3.640 internaçóes por outras gravidezes que terminaram em aborto, na Região Metropolitana de Porto Alegre. Percebe-se que o maior quantitativo de hospitalizações ocorreu em Porto Alegre, seguido de Canoas e Alvorada, perfazendo $42,06 \%, 10,47 \%$ e $10,11 \%$, respectivamente, conforme tabela 1. Nesses três municípios, considerando a população de cada local relacionando com número de internações que terminaram em aborto, mostra que Alvorada está em primeiro lugar com 0,19\% seguida de Canoas $0,12 \%$ e Porto Alegre $0,11 \%$.

Quanto a faixa etária, as maiores frequências de internações ocorreram por jovens adultas, principalmente na faixa etária de 20 a 29 anos correspondendo a 3.638 (41,40\%) das internações.

Já em relação a raça/cor prevaleceu as brancas 2.232 (64,32\%), sendo que em 971 registros, essa informação não foi apontada de acordo com a tabela 2. 
No que diz respeito ao caráter de atendimento, $3.260(89,56 \%)$ das mulheres internaram por urgência. $O$ tempo médio de permanência foi de 1,5 dias e o valor gasto pelo poder público, em todas as internações incluídas no período avaliado, foi de $\mathrm{R} \$ 706.650,84$. Apenas uma das hospitalizações teve como desfecho o óbito.

\section{DISCUSSÃO}

A partir da análise, percebe-se que em $1.551(42,6 \%)$ dos casos as hospitalizações ocorreram na capital. Pesquisas anteriores indicam que as mulheres optam por abortar em grandes capitais brasileiras a fim de preservar o anonimato e o risco de punições legais e também ao maior quantitativo de clínicas clandestinas ${ }^{12,13}$. Outros estudos baseados em hospitalizações do SIH/SUS sem diferenciação quanto ao CID-10 do aborto, contabilizaram 1.054.242 internações, com uma taxa média de 2,07 abortos por 100 mulheres ${ }^{1,14,15}$.

A faixa etária com maior hospitalização nessa pesquisa contemplou as idades entre 20 a 29 anos, corroborando com outros estudos que apontaram o mesmo ciclo etário $^{3,7}$. Devido ao ato ilegal do abortamento, os dados de abortos provocados foram subestimados, sendo classificados por diferentes maneiras de causas, ou seja, prejudicando também a avaliação da faixa etária. Além disso, foi concluído que apesar da obrigatoriedade de investigação das causas de óbito fetais e neonatais, apenas pouco mais da metade teria sido realizado ${ }^{16}$.

O maior número de hospitalização por abortamento se deu na raça/cor branca, representando $2.341(64,32 \%)$ da totalidade de internações, porém, de forma contrária, estudos anteriores observaram que a maior frequência de abortos ocorridos, ocorreram por mulheres de raça/cor pre$\mathrm{ta}^{8,17}$. Quanto ao tempo regular de internação, os achados se assemelham a estudos anteriores de 1,5 a 2,0 dias em média ${ }^{10,12,15}$, sugerindo uma internação pontual e breve para resolução de um aborto que inicia previamente à internação hospitalar.

Devido a indisponibilidade de dados quanto a escolaridade, estado civil, ocu- pação, a análise tornou-se limitada quanto ao cruzamento de dados sugestivos de vulnerabilidade social, entretanto, pesquisas prévias reportam que mulheres jovens, de baixa renda, com menor escolaridade, negras, sem companheiro e com maior número de filhos apresentaram maior prevalência de gestações não desejadas ${ }^{3,8}$.

\section{Os dados analisados}

apresentam uma

\section{amostra das}

hospitalizações por

outras gravidezes

\section{terminadas em}

\section{aborto. Tais dados}

representam uma alta

taxa considerando a

exclusão dos abortos

espontâneos e os

causados por razóes

\section{médicas.}

Quanto ao caráter de atendimento, as hospitalizações por urgência foram as mais frequentes 3.260 (89,56\%), indo ao encontro de pesquisa prévia em que os atendimentos de urgência estão em maior número8. Sugere-se que a maior ocorrência de hospitalizações em caráter de urgência, possa estar relacionada com o receio da procura de um atendimento hospitalar, devido ao medo da rejeição familiar e da sociedade, conflito em virtude de suas crenças e o risco de ser penalizada por um ato criminoso ${ }^{10,20}$.
A subnotificação associada ao fato da criminalização do aborto contribui para a omissão e consequente risco inerente ao ato do Código Penal, Artigos 126-129, Decreto-Lei no 2.848 de 7 de dezembro de 1.96914,21. Nos casos das mulheres em situação de abortamento, a qualidade dessa atenção deve ser realizada por uma postura ética, destituída de preconceitos e discriminações, oferendo proteção e o respeito aos direitos humanos 5 .

Em artigos publicados anteriormente, o mesmo traz a proporção de 1 mulher vítima de discriminação a cada 3 mulheres atendidas em situação de abortamento. Contudo, a ameaça de denúncias por parte dos profissionais de saúde para polícia, proibido por lei, esteve presente em todos os casos em que a mulher admitiu o uso de medicamentos ou métodos abortivos ${ }^{22,23}$.

É importante ressaltar que, segundo o Art. 128 do Código Penal - Decreto Lei 2848/40, o aborto é legal em caso de estupro e violência sexual não consentida, não necessitando de boletim de ocorrência policial provando o ocorrido. Se faz necessário maiores informações para os profissionais de saúde, que muitas vezes se recusam a fazê-lo por medo da proibição vigente no Brasil e que não se encaixa em casos de estupro, ou por motivos religiosos e éticos individuais ${ }^{22,23}$.

Há algumas limitações neste trabalho decorrentes da natureza administrativa do banco de dados SIH/SUS, os potenciais vieses decorrentes dessas limitações são reconhecíveis ainda que seja possível obter um panorama da situação das internações por outras gravidezes que terminam em aborto na RMPA de modo bastante ágil.

\section{CONCLUSÃO}

Os dados analisados apresentam uma amostra das hospitalizações por outras gravidezes terminadas em aborto. Tais dados representam uma alta taxa considerando a exclusão dos abortos espontâneos e os causados por razões médicas. Acredita-se que uma parte considerável dessas hospitalizações poderia ter sido evitada se o aborto não fosse considerado crime, 
uma atividade clandestina. A ilegalidade do aborto contribui para altas taxas de internação, evitáveis.

Torna-se necessário aprofundar o co- nhecimento desta realidade na Região Metropolitana de Porto Alegre, como por exemplo, o motivo pelo qual os abortos foram causados e as condiçôes sociodemográ- ficas destas mulheres que podem elucidar os fatores predisponentes. Pesquisas com a mesma temática devem ser estimuladas mesmo com todos os desafios implicados.

\section{REFERÊNCIAS}

1. Anjos, KF, Santos VC, Souzas R, Eugênio BG. Aborto e saúde pública no Brasil: reflexões sob a perspectiva dos direitos humanos. Saúde em Debate, Rio de Janeiro, 2013; 37(98): 504-15.

2. World Health Organization. Department of reproductive health and research. Safe abortion: technical and policy guindance for health systems. 2nd ed. Geneve (Swi): World Health Organization; 2012.

3. Diniz D, Medeiros M, Madeiro A. Pesquisa Nacional de Aborto 2016. Ciências \& Saúde Coletiva, Teresina; 2(22): 653-60.

4. Machado MRA, Cook RJ. Constitutionalizing abortion in Brazil. Rev. Investig. Const., v. 5, n. 3, p. 185-231, Curitiba, Dec.2018. Doi. org/10.5380/rinc.v5i3.60973

5. Brasil. Ministério da Saúde. Secretaria de Atenção à Saúde. Departamento de Ações Programáticas Estratégicas. Atenção humanizada ao abortamento: norma técnica / Ministério da Saúde, Secretaria de Atenção à Saúde, Área Técnica de Saúde da Mulher. - 2. ed. - Brasília: Ministério da Saúde, 2011.60 p. - (Série A. Normas e Manuais Técnicos) (Série Direitos Sexuais e Direitos Reprodutivos; Caderno $\left.n^{\circ} 4\right)$

6. Torres JHR. Aborto. Legislação comparada. Rev. Epos vol.2 no.2. RJ dez. 2011. http://pepsic.bvsalud.org/scielo.php?script=sci_arttext\&pid=S2178-700X2011000200005

7. Santos TF, Andreoni S, Silva RS. Prevalência e características de mulheres com aborto provocado - Favela México 70, São Vicente - São Paulo. Rev Bras Epidemiol, São Paulo, 2012; 15(1): 123-33.

8. Souza ZCSN, Diniz NMF. Aborto provocado: o discurso das muIheres sobre suas relações familiares. Texto \& Contexto - Enfermagem, 2011; 20(4): 742-50. FapUNIFESP (SciELO). http://dx.doi. org/10.1590/s0104-07072011000400013.

9. Silva MM, Castro PM, Theisen C, Freitas HMB, Colomé JS. Problemática Do Aborto Em Santa Maria, Rio Grande Do Sul: Dados Epidemiológicos. Disciplinarum Scientia: Série: Ciências da Saúde, Santa Maria, 2014; 15(2): 249-59.

10. Batista RQ, Koch DF, Bispo AMS, Luciano TV, Velten APC. Descrição das internações por aborto no estado do Espírito Santo, Brasil. Rev. Bras. Pesq. Saúde. Vitória, 2016; 2(18):79-86.

11. Brasil. Resolução № 510 de 07 de Abril de 2016. Disponível em: http://conselho.saude.gov.br/resolucoes/2016/Res0510.pdf

12. Cardoso BP, Vieira FMSB, Saraceni V. Aborto no Brasil: o que dizem os dados oficiais? Cad. Saúde Pública vol.36 supl.1 Rio de Janeiro 2020 Epub Feb 21, 2020.

13. Meneses GMS, Aquino EML, Fonseca SC, Domingues RMSM. Aborto e saúde no Brasil: desafios para a pesquisa sobre o tema em um contexto de ilegalidade. Cad. Saúde Pública 2020; 36 Sup 1:e00197918. doi: 10.1590/0102-311X00197918
14. Brasil. Ministério da Saúde. Secretaria de Ciência, Tecnologia e Insumos Estratégicos. Departamento de Ciência e Tecnologia. Aborto e saúde pública no Brasil: 20 anos / Ministério da Saúde, Secretaria de Ciência, Tecnologia e Insumos Estratégicos, Departamento de Ciência e Tecnologia. Brasilia: Ministério da Saúde, 2009.

15. Huffnpost, Brasil. Uma mulher morre a cada 2 dias por aborto inseguro. COFEN 2018. http://www.cofen.gov.br/uma-mulher-morre-a-cada-2-dias-por-causa-do-aborto-inseguro-diz-ministerio-da-saude_64714.html\#

16. Kale PL, Jorge MHPM, Fonseca SC, Cascão AM, Silva KS, Reis $A C$, et al. Mortes de mulheres internadas para parto e por aborto e de seus conceptos em maternidades públicas. Ciência \& Saúde Coletiva. 2018; 23(5):1577-90. FapUNIFESP (SciELO). http://dx. doi.org/10.1590/1413-81232018235.18162016.

17. Brasil. Ministério da Saúde. Protocolos da Atenção Básica: Saúde das Mulheres / Ministério da Saúde, Instituto Sírio-Libanês de Ensino e Pesquisa - Brasília: Ministério da Saúde, 2016. 230 p. : il. ISBN 978-85-334-2360-2

18. Adesse L, Silva KS, Bonan C, Fonseca VM. Complicações do abortamento e assistência em maternidade pública integrada ao Programa Nacional Rede Cegonha. Saúde em Debate, 2015; 39(106): 694-706. FapUNIFESP (SciELO). http://dx.doi. org/10.1590/0103-1104201510600030011.

19. Gesteira SMA, Barbosa VL, Endo PC. O luto no processo de aborto provocado. Acta Paulista de Enfermagem, 2006;19(4):4627. FapUNIFESP (SciELO). http://dx.doi.org/10.1590/s010321002006000400016.

20. Benute GRG, Nomura RMY, Pereira PP, Lucia MCSL, Zugaib M. Abortamento espontâneo e provocado: ansiedade, depressão e culpa. Revista da Associação Médica Brasileira, São Paulo, 2009; 55(3): 322-7.

21. Brasil. Ministério da Saúde. Secretaria de Políticas de Saúde. Área Técnica de Saúde da Mulher. Assistência em Planejamento Familiar: Manual Técnico/Secretaria de Políticas de Saúde, Área Técnica de Saúde da Mulher - 4 a edição - Brasilia: Ministério da Saúde, 2002150 p.: il. - (Série A. Normas e Manuais Técnicos; n.40)

22. Madeiro Alberto Pereira, Rufino Andréa Cronemberger. Maus-tratos e discriminação na assistência ao aborto provocado: a percepção das mulheres em Teresina, Piauí, Brasil. Ciênc. saúde coletiva [Internet]. 2017 Aug [cited 2020 May 04]; 22(8): 27712780. http://dx.doi.org/10.1590/1413-81232017228.04252016

23. Goes EF, Menezes GMS. Almeida MCC, Araújo TVB, Alves SV, Alves MTSSB, et al. Vulnerabilidade racial e barreiras individuais de mulheres em busca do primeiro atendimento pós-aborto. Cad. Saúde Pública [Internet]. 2020 [cited 2020 May 04] ; 36/ Suppl 1): e00189618. http://dx.doi.org/10.1590/0102-311x00189618. 